\title{
Spontaneously hypertensive rats are resistant to hypercholesterolaemia-induced atherosclerosis ${ }^{1}$
}

\author{
P.M. Pisulewski ${ }^{1,3}$, M. Franczyk ${ }^{1}$, R.B. Kostogrys ${ }^{1}$, B. Lorkowska ${ }^{2}$, \\ M. Bartuś ${ }^{2}$ and S. Chlopicki ${ }^{2}$
}

\author{
${ }^{1}$ Department of Human Nutrition, Faculty of Food Technology, \\ Agricultural University of Cracow \\ Balicka 122, 30-149 Kraków, Poland \\ ${ }^{2}$ Department of Experimental Pharamacology, Chair of Pharamacology, \\ Jagiellonian University Medical College \\ Grzegórzecka 16, 31-531 Kraków, Poland
}

(Received 1 July 2005; revised version 18 November 2005; accepted 6 January 2006)

\begin{abstract}
The objective of the present study was to re-evaluate critically the potential of spontaneously hypertensive rats (SHR) as models for diet-induced hypercholesterolaemia and the hypercholesterolaemia-induced atherosclerosis. Normotensive Wistar-Kyoto rats (WKY) were used as control animals. Twelve-months-old SHR $(n=22)$ and WKY $(n=17)$ male rats, were randomly divided into two groups each and fed one of the following diets for 12 consecutive weeks: the control diet i.e. the basal AIN-93M diet and the hypercholesterolaemic diet (g/100g: cholesterol 1, cholic acid 0.5 and butter 20). The hypercholesterolaemic diet increased highly significantly $(\mathrm{P}<0.001)$ serum total cholesterol concentrations in both WKY (control vs high-cholesterol) and SHR (control vs high cholesterol) rats $(2.59 \pm 0.20$ vs $6.54 \pm 0.33$ and $2.52 \pm 0.20$ vs $6.64 \pm 0.89 \mathrm{mmol} / \mathrm{L}$, respectively). The same was true for LDL-cholesterol $(0.75 \pm 0.09$ vs $4.22 \pm 0.26$ and $0.84 \pm 0.05$ vs $4.62 \pm 0.69 \mathrm{mmol} / \mathrm{L}$, respectively). HDL-cholesterol concentrations were also moderately increased in cholesterol-fed rats $(1.46 \pm 0.10$ vs $1.95 \pm 0.09$ and $1.24 \pm 0.08$ vs $1.53 \pm 0.17 \mathrm{mmol} / \mathrm{L}$, respectively) whereas triglicerydes were unaffected. As regards lipid profile, the only effect of animal strain was that noted for SHR rats in which HDL cholesterol concentrations were significantly $(\mathrm{P}<0.05)$ lower than in WKY rats $(1.39 \pm 0.10$ vs $1.69 \pm 0.09 \mathrm{mmol} / \mathrm{L}$, respectively). Serum concentrations of MCP-1 were elevated in both WKY (control vs high-cholesterol) and SHR rats (control vs high cholesterol, $41.22 \pm 7.98$ vs $156.10 \pm 20.66$ and $84.88 \pm 49.40$ vs $181.65 \pm 38.40 \mathrm{pg} / \mathrm{mL}$, respectively) though

\footnotetext{
${ }^{1}$ Supported by the State Committee for Scientific Research, Grants No. 3-P06T-029-24, 3-P05A-003-25, PBZ-KBN-101/T09/2003/6 and by Professorial Grant from The Foundation for Polish Science (SC)

${ }^{3}$ Corresponding author: e-mail: rrpisule@cyf-kr.edu.pl
} 
only in WKY rats this increase reached statistical significance. Either in WKY or SHR rats, the hypercholesterolaemic diet had no significant effect on endothelium-dependent nor endotheliumindependent vasodilation in aorta induced by acetylcholine or SNAP, respectively. Histological examination of proximal aortas from WKY (control vs high-cholesterol) and SHR (control vs highcholesterol) rats did not show any structural changes, indicative of atherosclerotic plaque formation. We conclude that diet induced hypercholesterolaemia does not lead to progression of atherosclerosis in SHR rats. Hence, hypertensive rats, fed hypercholesterolaemic diet, are not appropriate models for human hypercholesterolaemia and atherosclerosis.

KEY WORDS: spontaneous hypertensive rats (SHR), hypercholesterolaemia, inflammation, endothelial functions, atherosclerosis

\section{INTRODUCTION}

We have recently reported that diet-induced hypercholesterolaemia did not impair endothelial vascular functions and did not lead to development of arterial atherosclerotic lesions in normotensive Wistar rats (Pisulewski et al., 2005). We also indicated, that the diet-induced hypercholesteroleamia did not result in an endothelial inflammatory response, a key factor in the initiation of atherosclerosis in humans (Ross, 1999; Hansson, 2005). Hence, according to our findings, normotensive Wistar rat, cannot be recommended as reliable animal model for studies on potential of human foods or diets to modify hypercholesterolaemia-induced atherosclerosis.

At the same time it is known that hypercholesterolaemia is not only one of possible causes of endothelial dysfunction leading to atherosclerosis. In humans, classical risk factors of atherosclerosis include also hypertension, diabetes, hyperhomocysteinaemia, aging, and life-style factors such as physical inactivity and smoking (Chłopicki and Gryglewski, 2004; Guzik et al., 2004; Kitamoto and Egashira, 2004). In view of the complexity of risk factors leading to development of atherosclerosis, we decided to include in our study an extra variable of this disease, notably hypertension. Indeed, vascular endothelial functions were impaired in hypertensive animal models and humans and this impairment resulted finally in atherosclerosis (e.g., Herrera et al., 2001; Vasconen et al., 2002).

The objective of the present study was to develop an animal model suitable for studies on functional i.e. hypocholesterolaemic and/or antiatherogenic properties of human foods or diets. Since both hypertension and hypercholesterolaemia can impair vascular functions, we investigated the effects of combination of systemic hypertension and diet-induced hypercholesterolaemia on development of atherosclesoris in rats. We evaluated serum lipid profile, markers of inflammatory response, endothelial functions and development of aortic atherosclerotic lesions in normotensive Wistar-Kyoto (WKY) and spontaneously hypertensive rats (SHR). Our approach was similar to that described by Cappelli-Bigazzi et al. (1997). 


\section{MATERIAL AND METHODS}

The experimental procedures were conducted according to the Guidelines for Animal Care and Treatment of the European Community and were approved by the Local Animal Ethics Commission.

\section{Animals and feeding}

Twenty months-old SHR $(\mathrm{n}=22)$ and WKY $(\mathrm{n}=17)$ male rats, weighing 350 $-550 \mathrm{~g}$ were obtained from the Animal Laboratory of the Polish Mother's Health Centre Research Institute in Łódź (Poland). The rats were housed in group of 5 in plastic cages, in an isolated room at controlled temperature and ambient humidity. Lights were maintained on a $12 \mathrm{~h}$ light-dark cycle. The animals were acclimatized to these conditions for 1 week and given free access to water and the basal semipurified AIN-93M diet (Reeves et al., 1993). After the adaptation period, both SHR and WKY rats were randomly divided into two groups. Each group was then fed one of the following diets for 12 consecutive weeks: 1 . the control diet i.e. the basal AIN-93M diet and 2. the hypercholesterolaemic diet. The latter diet was the basal AIN-93M diet supplemented with: cholesterol 1g/100 g (Sigma, St. Louis, MO), cholic acid $0.5 \mathrm{~g} / 100 \mathrm{~g}$ (Sigma, St. Louis, MO) and butter $20 \mathrm{~g} / 100 \mathrm{~g}$. The final composition of the diets is given in Table 1 .

Table 1. Composition of experimental diets based on the AIN93M (Reeves et al., 1993) formulation, $\mathrm{g} / \mathrm{kg}$ diet

\begin{tabular}{lcc}
\hline Ingredient & Control & Hypercholesterolaemic \\
\hline Maize starch & 622.49 & 447.49 \\
Casein $(95 \% \mathrm{~N} \times$ 6.25) & 140.00 & 140.00 \\
Sucrose & 100.00 & 100.00 \\
Soyabean oil & 40.00 & - \\
Fibre $^{1}$ & 50.00 & 50.00 \\
Mineral mix $^{2}$ & 35.00 & 35.00 \\
${\text { Vitamin } \text { mix }^{3}}_{\text {Butter }}$ & 10.00 & 10.00 \\
Choline bitartrate $^{4}$ & - & 200 \\
Tert-butylhydroquinone $^{5}$ & 2.50 & 2.50 \\
Cholesterol $^{6}$ & 0.008 & 0.008 \\
Cholic acid $^{7}$ & - & 10.00 \\
\hline
\end{tabular}

${ }^{1}$ Alpha cellulose powder - Sigma (St. Louis, MO, USA); ${ }^{2}$ AIN-93M-MX (Reeves et al., 1993); ${ }^{3}$ AIN93M-VX (Reeves et al., 1993); ${ }^{4.5}$ Sigma (St. Louis, MO, USA); ${ }^{6}$ Aldrich (Steinheim, Gemany); ${ }^{7}$ Fluka (Buchs, Switzerland)

Indirect blood pressure (BP) was monitored by the tail-cuff method at the beginning of the experiment and after 12 weeks of the experimental period. Mean 
BP were significantly higher in the SHR than in the control WKY rats $(189 \pm 6.7$ $\mathrm{mmHg}$ vs $119 \pm 10.4 \mathrm{mmHg}$, respectively).

Determination of serum lipid profile and markers of inflammation (WKY vs SHR rats)

After 12 weeks of feeding with the respective diets, rats were deprived of food overnight, anesthetized intraperitoneally with sodium thiopental (120-150 mg kg ${ }^{-1}$ body weight) (Biochemie $\mathrm{GmbH}$, Kundl-Rakusko, Austria) and killed by withdrawing blood from the heart. The blood samples were collected in test tubes and centrifuged $(4000 \mathrm{~g}, 10 \mathrm{~min})$ to obtain serum. Samples of serum were immediately frozen $\left(-20^{\circ} \mathrm{C}\right)$ and stored until analysis. They were analysed using commercially available kits for total cholesterol (OSR 6116), HDL-cholesterol (OSR 6156) and triacylglycerols (OSR 6133); Olympus Diagnostica GmbH, Hamburg (Germany). The concentration of LDL-cholesterol was calculated according to the formula of Friedewald et al. (1972). The lipoprotein concentrations were expressed in $\mathrm{mmol} / \mathrm{L}$.

Serum concentrations of an inflammatory chemokine i.e. monocyte chemoattractant protein-1 (MCP-1) were analysed in previously stored $\left(-70^{\circ} \mathrm{C}\right)$ serum samples using the commercially available enzyme immunoassay kits (BioSource, Camarillo, California, USA). They were expressed in $\mathrm{pg} / \mathrm{mL}$.

\section{Assessment of vascular endothelial functions (WKY vs SHR rats)}

After 12 weeks of feeding with the respective diets, rats were euthanized by intraperitoneal thiopental injection (120-150 mg kg-1 body weight) (Biochemie $\mathrm{GmbH}$, Kundl-Rakusko, Austria). The aortas were rapidly removed by central sternotomy, washed out with ice-cold saline, and placed in Krebs-Hanseleit solution. After careful removal of the surrounding adventitial tissue, the aortas were cut into six rings, 3-4 $\mathrm{mm}$ in length. All experiments were performed on aortic rings with endothelium as previously described (Pisulewski et al., 2005). Maximal contractile response was evoked by potassium chloride $(\mathrm{KCl}, 30-90$ $\mathrm{mM}$ ), then a submaximal contraction (approximately $60-80 \%$ of maximal $\mathrm{KCl}$ induced response) was induced by an $\alpha_{1}$-adrenergic agonist - phenylephrine $\left(10^{-8}\right.$ $-3 \times 10^{-7} \mathrm{M}$ ). Endothelial functions were assessed by cumulative concentrationdependent responses to an endothelium-dependent vasodilator - acetylcholine $\left(10^{-9}-10^{-5} \mathrm{M}\right)$. Moreover, an endothelium-independent vasodilator - S-nitroso-Nacetyl-penicillamine (SNAP: $10^{-9}-10^{-5} \mathrm{M}$ ), was used to test smooth muscle cell functions. Experiments on aortic rings derived from the WKY or SHR control rats (3 rings per rat) and the WKY or SHR rats fed with the high-cholesterol diet (3 
rings per rat) were done simultaneously. All relaxation results are expressed as a percentage of the maximal precontraction of phenylephrine-induced responses.

Tissue preparation and histological examination of atherosclerosis (WKY vs SHR rats)

The present procedures were performed as described before (Pisulewski et al., 2005). The dissected proximal aortas (after 12 weeks feeding, $n=2$ ) were gently washed to remove blood and fixed immediately in $10 \%$ buffered formalin ( $\mathrm{pH}$ 7.4) for about $24 \mathrm{~h}$ at room temperature. They were washed again for $2 \mathrm{~h}$, dehydrated through a graded ethanol series, placed in xylene (3 times for $15 \mathrm{~min}$ ), embedded in paraffin $\left(58^{\circ} \mathrm{C}, 3 \mathrm{~h}\right)$ and cast into blocks. Tissue samples were cut into approximately $5 \mu \mathrm{m}$-thick sections by microtome, placed on glass slides and stained by hematoxylin and eosin. Serial sections of the proximal aorta starting from the aortic root up to the bifurcation of carotid artery were examined. Light microscopic examination and photographic documentation were performed using a Zeiss microscope connected to a digital camera (Olympus).

\section{Statistical analysis}

Results are expressed as means \pm SEM. Where appropriate, the data were subjected to two-way analysis of variance to test for differences among experimental treatments including animal strain (WKY vs SHR) and type of diet (control vs high-cholesterol), and to identify possible interactions between experimental treatments. The analysis was generated by STATISTICA version 6.1 package (StatSoft, Inc., USA). The differences between treatments and corresponding interactions were identified by the $\mathrm{F}$ test at $\mathrm{P}<0.05$ and $\mathrm{P}<0.01$ significance levels.

\section{RESULTS}

Serum lipid profile and inflammatory responses in WKY and SHR rats

The high-cholesterol diet resulted in an increase in serum total cholesterol concentrations in both WKY (control vs high-cholesterol) and SHR (control vs high cholesterol) rats $(2.59 \pm 0.20$ vs $6.54 \pm 0.33$ and $2.52 \pm 0.20$ vs $6.64 \pm 0.89 \mathrm{mmol} / \mathrm{L}$, respectively $(\mathrm{P}<0.001)$. The same was true for LDL-cholesterol $(0.75 \pm 0.09$ vs $4.22 \pm 0.26$ and $0.84 \pm 0.05$ vs $4.62 \pm 0.69 \mathrm{mmol} / \mathrm{L}$, respectively; $\mathrm{P}<0.05)$. HDLcholesterol concentrations were also elevated in rats fed high-cholesterol diet ( $1.46 \pm 0.10$ vs $1.95 \pm 0.09$ and $1.24 \pm 0.08$ vs $1.53 \pm 0.17 \mathrm{mmol} / \mathrm{L}$, respectively; $\mathrm{P}<0.05$ ) 
whereas triacylglycerols were unaffected (Figures 1A and 1B). As regards lipid profile, the only effect of animal strain was that noted for SHR rats in which HDL cholesterol concentrations were significantly $(\mathrm{P}<0.05)$ lower than in WKY rats $(1.39 \pm 0.10$ vs $1.69 \pm 0.09 \mathrm{mmol} / \mathrm{L}$, respectively). No significant interactions between treatments were detected.

$\mathbf{A}$

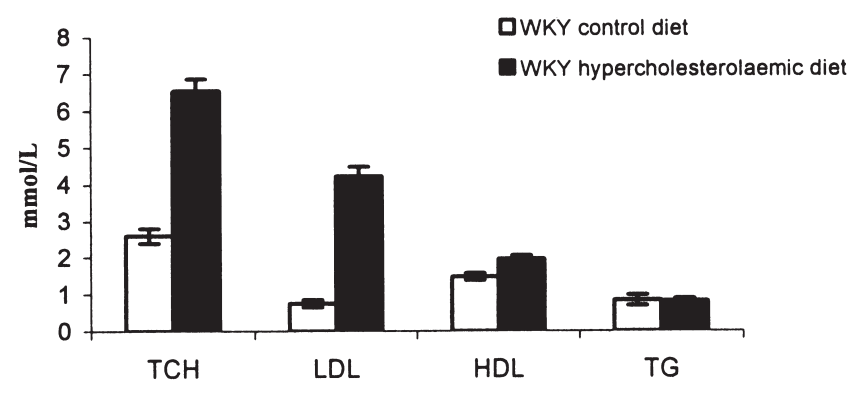

B

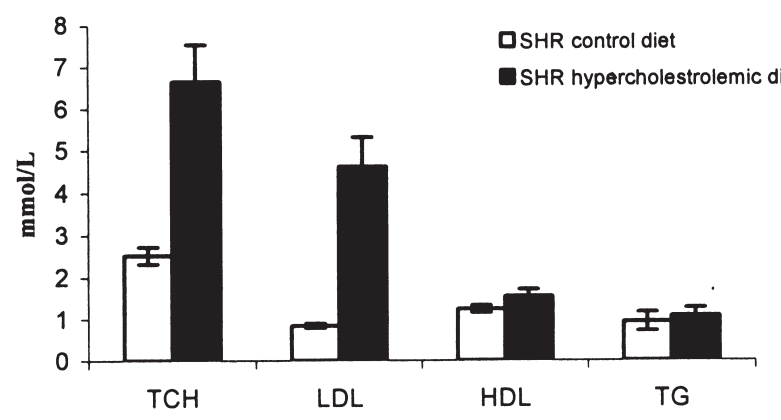

Figure 1. A. Serum levels of total cholesterol, LDL-cholesterol, HDL-cholesterol and triglycerides in WKY rats fed the control and the hypercholesterolaemic diet for 12 weeks. Values are means \pm SEM. B. Serum levels of total cholesterol, LDL-cholesterol, HDL-cholesterol and triglycerides in SHR rats fed the control and the hypercholesterolaemic diet for 12 weeks. Values are means $\pm \mathrm{SEM}$

Serum concentrations of an inflammatory MCP-1 were moderately elevated in both WKY (control vs high-cholesterol) and SHR (control vs high cholesterol) rats $(41.22 \pm 7.98$ vs $156.10 \pm 20.66 ; \mathrm{P}<0.01)$ and $84.88 \pm 49.40$ vs $181.65 \pm 38.40$ $\mathrm{pg} / \mathrm{mL}$, respectively) (Figure 2). They also tended to be higher in SHR rats than in WKY rats. However, the latter effect was insignificant. As above, there were no significant interactions between treatments. 


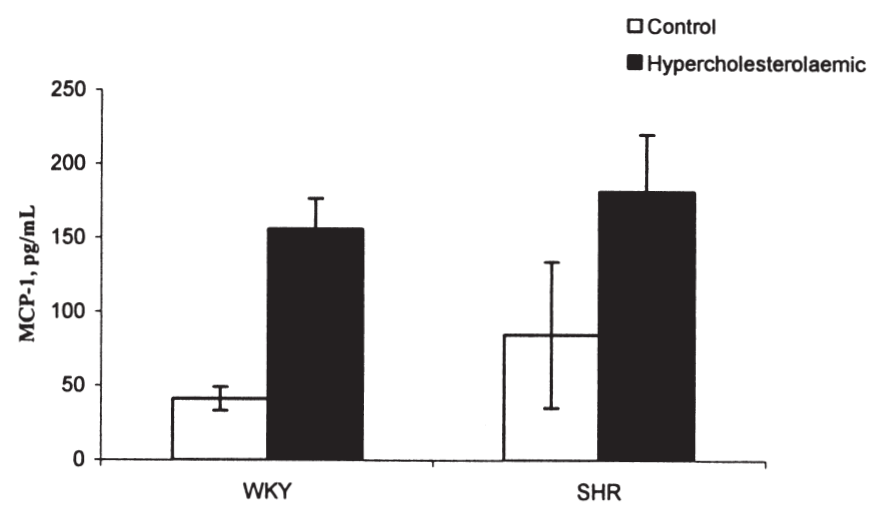

Figure 2. Serum levels of MCP-1 in WKY and SHR rats fed the control and the hypercholesterolaemic diet for 12 weeks. Values are means \pm SEM

Effects of hypercholesterolaemic diets on endothelium-dependent and endotheliumindependent vascular responses in WKY and SHR rats

Relaxation responses to acetylcholine (10-9-10-5M) and S-nitroso-N-acetylpenicillamine (SNAP; $10-9-10-5 \mathrm{M}$ ) in aorta were expressed as the percent change relative to the constriction induced by phenylephrine. The experimental treatments had no significant effect on endothelium-dependent vascular relaxation responses induced by acetylcholine (Figure 3A). These responses were comparable over the range of acetylcholine concentrations, regardless of the type of diet and the animal strain. The maximal vasorelaxant responses to acetylcholine for WKY (control vs high-cholesterol diet) and SHR (control vs high-cholesterol diet) rats were noted at the concentration of $10-5 \mathrm{M}(70.85 \pm 3.84 ; 77.0 \pm 4.0 ; 75.36 \pm 5.27$ and $73.81 \pm 4.9 \%$, respectively).

In the same way, vascular relaxation responses induced by an endotheliumindependent vasodilator were not affected by the experimental treatments. As above, the maximal vasorelaxat responses to SNAP were observed at the concentration of $10^{-5} \mathrm{M}$ and reached approximately $100 \%$ (Figure $3 \mathrm{~B}$ ).

Effects of hypercholesterolaemic diets on development of atherorosclerotic lesions in WKY and SHR rats

Histological examination of proximal aortas from WKY (control vs highcholesterol) and SHR (control vs high-cholesterol) rats did not show any structural changes, indicative of atherosclerotic plaque formation (Figures 4A, 4B, 4C and $4 \mathrm{D}$, respectively). Endothelium, intima and adventitia of the examined vessels were unchanged. 


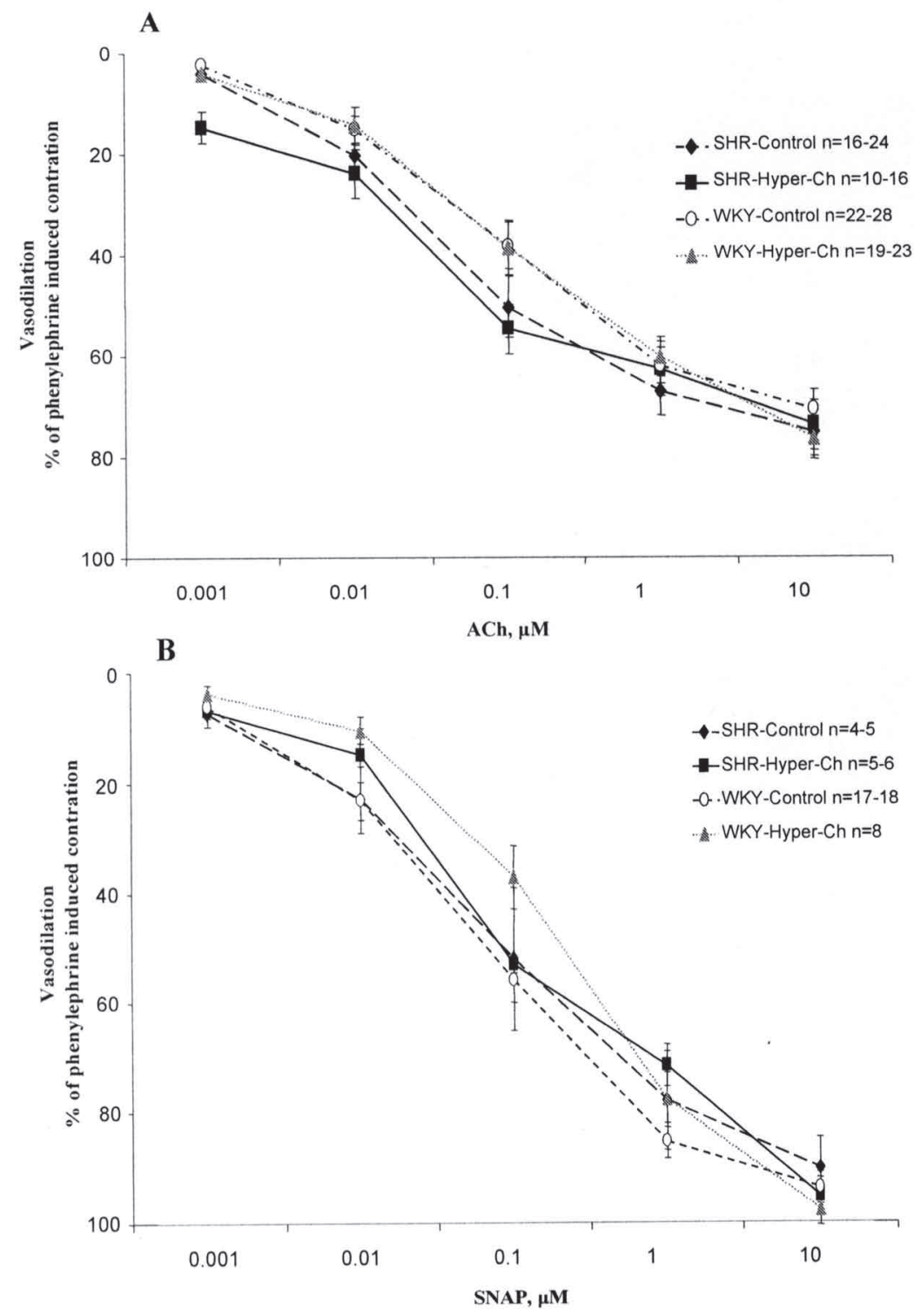

Figure 3. A. Cumulative concentration-dependent relaxation responses to acetylcholine, expressed as a percentage of phenylephrine-induced precontraction of isolated endothelium-intact arterial rings, from WKY and SHR rats fed the control and the hypercholesterolaemic diet for 12 weeks. Values are means \pm SEM. B. Cumulative concentration-dependent relaxation responses to SNAP, expressed as a percentage of phenylephrine-induced precontraction of isolated endothelium-intact arterial rings, from WKY and SHR rats fed the control and the hypercholesterolaemic diet for 12 weeks. Values are means \pm SEM 
$\mathbf{A}$

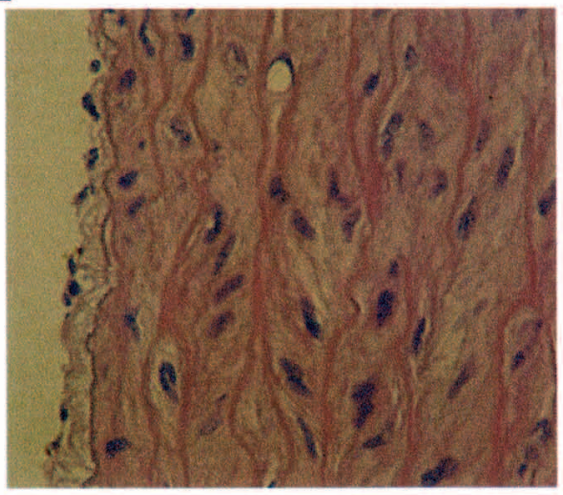

C

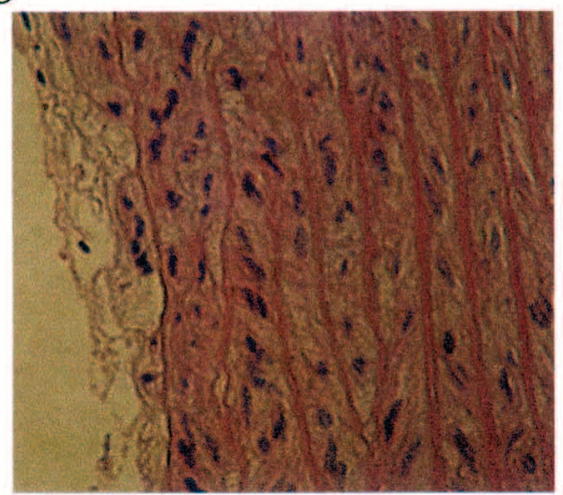

B

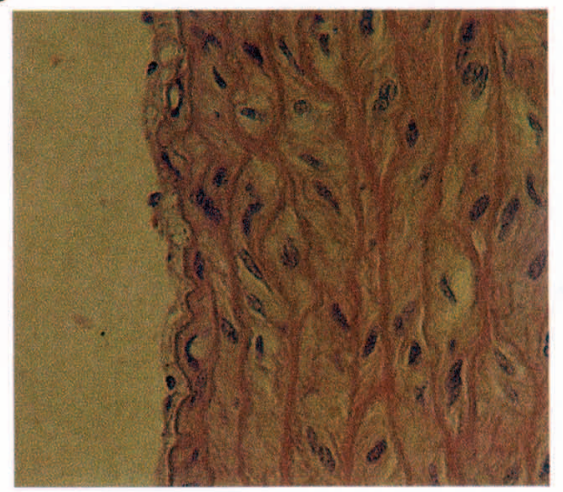

D

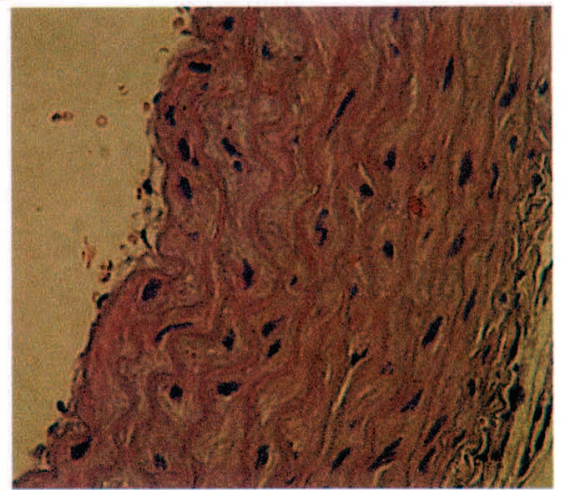

Figure 4. Light microscopy photographs of cross sections of the aortas, stained with hematoxylin and eosin, from WKY rats fed the control (A) and the hypercholesterolaemic (B) diet and from SHR rats fed the control (C) and the hypercholesterolaemic (D) diet for 12 weeks $(200 \mathrm{x})$

\section{DISCUSSION}

As indicated above (see Introduction), hypertension has been reported to induce endothelial dysfunction in humans and to be one of established risk factors for atherosclerosis (Chłopicki and Gryglewski, 2004; Guzik et al., 2004; Kitamoto and Egashira, 2004). Therefore, several attempts have been made to use genetically hypertensive animals, e.g., spontaneously hypertensive rats (SHR), as models for human atherosclerosis (Cheng and Shibata, 1981; Shirasaki et al., 1988; Sunano et al., 1989). More recently, an animal model involving the combination of systemic hypertension and hypercholesterolaemia was proposed (Cappelli-Bigazzi et al., 1997). At the same time, Cappelli-Bigazzi et al. (1997), did not provide 
histological evidence of the presence of vascular atherosclerotic lesions, to support that hypercholesterolaemic SHR rats develop artherosclerosis. In contrast, the obese Zucker rats, fed an atherogenic diet, have been successfully used as animal models for human metabolic syndrome (Vasconen et al., 2002). In these studies, feeding high-cholesterol diet resulted in impairment of vascular endothelium-dependent functions. More importantly, feeding the above diet led to evident development of atherosclerotic lesions in coronary heart and kidney arteries.

Since it is evident that suitable animal models of human diseases (e.g., CVD) are urgently needed to verify functional claims, made for functional foods considered to have beneficial health-related effects (Herrera et al., 2001; Muller et al., 2003; Zhao et al., 2004; Hilpert at al., 2005), we evaluated the potential of SHR rats as models for both hypercholesterolaemia and atherosclerosis. In line with our previous studies using normotensive Wistar rats (Pisulewski et al., 2005), we demonstrated again that cholesterol-fed normotensive (WKY) and hypertensive rats (SHR), developed comparable levels of severe hypercholesterolaemia. Total serum cholesterol LDLcholesterol and HDL-cholesterol concentrations fraction followed the same pattern. Less pronounced changes were noted for concentrations of triglycerides (Figures 1A and 1B). Hence, we were able to induce an atherogenic lipoprotein profile in both WKY and SHR rats. This view is supported even further by lower HDL-cholesterol concentrations in SHR rats. In general, the above findings are comparable with the results of similar experiments (Cappelli-Bigazzi et al., 1997; Yuan et al., 1998).

It is noteworthy, that hypercholesterolaemia in WKY or SHR rats resulted in elevation of serum concentration of a proinflammatory chemokine monocyte chemoattractant protein-1 (MCP-1). Indeed, inflammatory cytokines, including MCP-1, vascular cell adhesion molecule-1 (VCAM-1) and intracellular adhesion molecule-1 (ICAM-1) are considered to be markers involved in development of atherosclerosis. Importantly, MCP-1 was indicated as a maker of early inflammation, mediating monocyte attachment to the endothelium and diapedesis into the subendothelial space (Kataoka et al., 2002). In the present study, both WKY and SHR cholesterol-fed rats showed elevated MCP-1 serum concentrations relative to rats fed the control diet (Figure 2). Consequently, the elevated MCP-1 concentrations might be indicative of an early stage of endothelial dysfunction and atherosclerotic lesion formation. Surprisingly enough, the combination of systemic hypertension and hypercholesterolaemia in WKY and SHR rats did not produce any changes in vascular relaxation responses neither to acetylcholine nor to SNAP (Figures 3A and 3B). In contrast to our findings, CappelliBigazzi et al. (1997) demonstrated that feeding normotensive (WKY) and hypertensive (SHR) rats with an atherogenic (high-cholesterol) diet, led to impaired endotheliumdependent vascular relaxation to acetylcholine; more pronounced in SHR strain. Interestingly, endothelium-independent vascular relaxation to nitro-glycerine was not impaired in WKY rats whereas SHR rats showed evident vascular dysfunction. The 
discrepancy between results are not clear, since the increase in total cholesterol and LDL-cholesterol concentrations by hypercholesterolaemic diet in SHR or WKY rats reported by us and by Cappelli-Bigazzi et al. (1997) were comparable. Furthermore, we used 12 weeks experimental period of hypercholesterolaemic diet, while CappelliBigazzi et al. (1997) and Vasconen et al. (2002) used 4-week experimental periods in their studies.

Taking all together, in our hand, 12-week long hypercholesterolaemic diet either in SHR or in WKY rats did not lead to the impairment of NO-mediated function and robust inflammatory response (Zhang et al., 2004). No doubt, atherogenesis is an inflammatory disease, and the pronounced inflammatory response of endothelium is an essential prerequisite for its dysfunction and formation of atherosclerotic lesions (Hansson, 2005). Since it was not the case, we were not able to demonstrate any structural changes, indicative of atherosclerotic plaque formation, in proximal aortas from WKY and SHR rats fed high-cholesterol diets (Figures 4A, 4B, 4C and 4D). Endothelium, intima and adventitia were unchanged.

In view of the above findings a laboratory rat model for human atherosclerosis is invalid. Instead, we strongly support the use of mouse models of human atherosclerosis, both apolipoproteinE-deficient(knockout) mice and LDL receptor-deficient (knockout) mice. Recently, new combined apolipoprotein E-deficient and LDL receptor-deficient (double-knockout) mice have been introduced (Jawień et al., 2004).

\section{CONCLUSIONS}

Laboratory rats, both normotensive and hypertensive, fed high-cholesterol diets, develop severe hypercholesterolaemia. However the diet-induced hypercholestrolaemia does not lead to impairment of endothelial vascular functions, inflammatory responses and development of atherosclerotic lesions. Thus, we confirm the opinion that laboratory rats, including SHR strain, are not reliable models for human atherosclerosis (Moghadasian, 2002). Consequently, their resistance to atherosclerosis, limits the use of laboratory rats to verify functional (i.e. anti-atherogenic) effects of different dietary interventions. Instead we strongly support the use of apolipoprotein E-deficient or LDL receptor-deficient mice.

\section{REFERENCES}

Cappelli-Bigazzi M., Rubattu S., Battaglia C., Russo R., Enea I., Ambrosio G., Chiariello M., Volpe M., 1997. Effects of high-cholesterol and atherogenic diets on vascular relaxation in spontaneously hypertensive rats. Amer. J. Physiol. 273, H647-H654

Cheng J.B., Shibata S., 1981. Vascular relaxation in the spontaneously hypertensive rat. J. Cardiovasc. Pharmacol. 3, 1126-1140 


\section{HYPERCHOLESTEROLAEMIA-INDUCED ATHEROSCLEROSIS IN RATS}

Chłopicki S., Gryglewski R.J., 2004. Endothelial dysfunction in diabetes - a risk factor of atherothrombosis (in Polish). In: M. Tendera, K. Kawecka-Jaszcz, D. Czarnecka (Editors). Diabetes and Heart. Via Media, Gdańsk, pp. 1-19

Friedewald W.T., Levy R.I., Fredrickson D.S., 1972. Estimation of the concentration of low-density lipoprotein cholesterol in plasma, without use of the preparative centrifuge. Clin. Chem. 18, 499-502

Guzik T.J., Adamek-Guzik T., Jopek A., Korbut R., 2004. Pathological mechanisms of vascular endothelial dysfunction in atherosclerosis (in Polish). Czynniki Ryzyka 1-2, 8-22

Hansson G.K., 2005. Inflammation, atherosclerosis and coronary artery disease. N. Engl. J. Med. $352,1685-1695$

Herrera M.D., Perez-Guerrero C., Marhuenda E., Ruiz-Gutierrez V., 2001. Effects of dietary oleicrich oils (virgin olive and high-oleic-acid sunflower) on vascular reactivity in Wistar-Kyoto and spontaneously hypertensive rats. Brit. J. Nutr. 86, 349-357

Hilpert K.F., Kris-Etherton P.M., West S.G., 2005. Lipid response to a low-fat diet with or without soy is modified by C-reactive protein status in moderately hypercholesterolemic adults. J. Nutr. $135,1075-1079$

Jawień J., Nastalek P., Korbut R., 2004. Mouse models of experimental atherosclerosis. J. Physiol. Pharmacol. 55, 503-517

Kataoka C., Egashira K., Inoue S., Takemoto M., Ni W., Koyanagi M., Kitamoto S., Usui M., Kaibuchi K., Shimokawa H., Takeshita A., 2002. Important role of Rho-kinase in the pathogenesis of cardiovascular inflammation and remodeling induced by long-term blockade of nitric oxide synthesis in rats. Hypertension 39, 245-250

Kitamoto S., Egashira K., 2004. Endothelial dysfunction and coronary atherosclerosis. Curr. Drug Targets Cardiovasc. Haematol. Disord. 4, 13-22

Moghadasian M.H., 2002. Experimental atherosclerosis: a historical overview. Life Sci. 70, 855865

Muller H., Lindman A.S., Brantsaeter A.L., Pedersen J.I., 2003. The serum LDL/HDL cholesterol ratio is influenced more favorably by exchanging saturated with unsaturated fat than by reducing saturated fat in the diet of women. J. Nutr. 133, 78-83

Pisulewski P.M., Kostogrys R.B., Lorkowska B., Bartuś M., Chłopicki S., 2005. Critical evaluation of normotensive rats as models for hypercholesterolaemia-induced atherosclerosis. J. Anim. Feed Sci. 14, 333-345

Reeves P.G., Nielsen F.H., Fahey G., 1993. AIN-93 purified diets for laboratory rodents: final report of the Amrican Institute of Nutrition ad hoc writing committee on the reformulation of the AIN76 rodent diet. J. Nutr. 123, 1939-1951

Ross R., 1999. Atherosclerosis-an inflammatory disease. N. Engl. J. Med. 340, 115-126

Shirasaki Y., Kolm P., Nickols G.A., Lee T.J., 1988. Endothelial regulation of cyclic GMP and vascular responses in hypertension. J. Pharmacol. Exp. Ther. 245, 53-58

Sunano S., Osugi S., Shimamura K., 1989. Blood pressure and impairment of endothelium-dependent relaxation in spontaneously hypertensive rats. Experientia 45, 705-708

Vasconen T., Mervaala E., Krogerus L., Karppanen H., 2002. Suplementation of plant sterols and minerals benefits obese Zucker rats fed an atherogenic diet. J. Nutr. 132, 231-237

Yuan Y.V., Kitts D.D., Godin D.V., 1998. Variations in dietary fat and cholesterol intakes modify antioxidant status of SHR and WKY rats. J. Nutr. 128, 1620-1630

Zhao G., Etherton T.D., Martin K.R., West S.G., Gillies P.J., Kris-Etherton P.M., 2004. Dietary alphalinolenic acid reduces inflammatory and lipid cardiovascular risk factors in hypercholesterolemic men and women. J. Nutr. 134, 2991-2997

Zhang R., Ma J., Xia M., Zhu H., Ling W.H., 2004. Mild hypercholesterolemia induced by feeding rats diets rich in methionine or deficient in folate promotes early atherosclerotic inflammatory process. J. Nutr. 134, 825-830 